\title{
On Sparse Maximal 2-Planar Graphs
}

\author{
Christopher Auer, Franz J. Brandenburg, \\ Andreas Gleißner, and Kathrin Hanauer \\ University of Passau, 94030 Passau, Germany \\ $\{$ auerc, brandenb, gleissner, hanauer $\} @$ fim. uni-passau.de
}

A simple undirected graph $G=(V, E)$ is $k$-planar if it can be drawn in the plane such that each edge is crossed at most $k$ times, incident edges do not cross, and a pair of edges must not cross twice. Such graphs have attracted many graph drawers, see [1] and the references given there.

A drawing of a graph implies an embedding, which in addition to the vertices and edges specifies the faces. An embedding is $k$-planar if it is obtained from a $k$-planar drawing. Each $k$-planar drawing or embedding of a graph implies a rotation system, which is given by the clockwise order of the incident edges at each vertex. A rotation system is $k$-planar if it admits a $k$-planar drawing. The terms rotation system and embedding are often identified for planar graphs. However, they can differ for $k$-planar graphs with $k \geq 1$ since a $k$-planar rotation system does not necessarily determine the crossing edges, and a rotation system and the crossing edges do not determine the embedding. Finally, a graph is $k$-planar if it has a $k$-planar drawing.

In [1], we have considered maximal 1-planar graphs. Here1, we consider 2planarity on four levels of specification: Embeddings, rotation systems together with the set of pairs of crossing edges, rotation systems, and graphs. We distinguish these levels with respect to maximality, which means that the addition of any edge violates 2 -planarity.

At GD96 Pach and Toth have shown that 2-planar graphs with $n$ vertices have at most $5 n-10$ edges and this bound is tight. However, there are maximal 1-planar graphs [1] and 2-planar graphs which are even sparser than maximal planar graphs. We can establish the following bounds on the lowest density.

Theorem 1. For arbitrarily large $n$ there are

- maximal 2-planar embeddings with $\frac{4}{3} n+\mathcal{O}(1) \approx 1.33 n$ edges,

- maximal 2-planar rotation systems together with a set of pairs of crossing edges with $\frac{4}{3} n+\mathcal{O}(1) \approx 1.33 n$ edges,

- maximal 2-planar rotation systems with $\frac{7}{4} n+\mathcal{O}(1) \approx 1.75 n$ edges, and

- maximal 2-planar graphs with $\frac{387}{147} n+\mathcal{O}(1) \approx 2.63 n$ edges.

For these bounds we construct 2-planar graphs (with a particular embedding or a fixed rotation system) which include many so-called hermits. Hermits are vertices of degree 1 or 2 which are enclosed by crossing edges and cannot be connected to other vertices. For the density of $\frac{387}{147} n \approx 2.63 n$ of maximal 2-planar graphs consider the crossed fullerene $X C_{60}$, as shown in Fig. 1, It is obtained

${ }^{1}$ http://www.infosun.fim.uni-passau.de/br/publications/gd12-2p-poster.pdf 


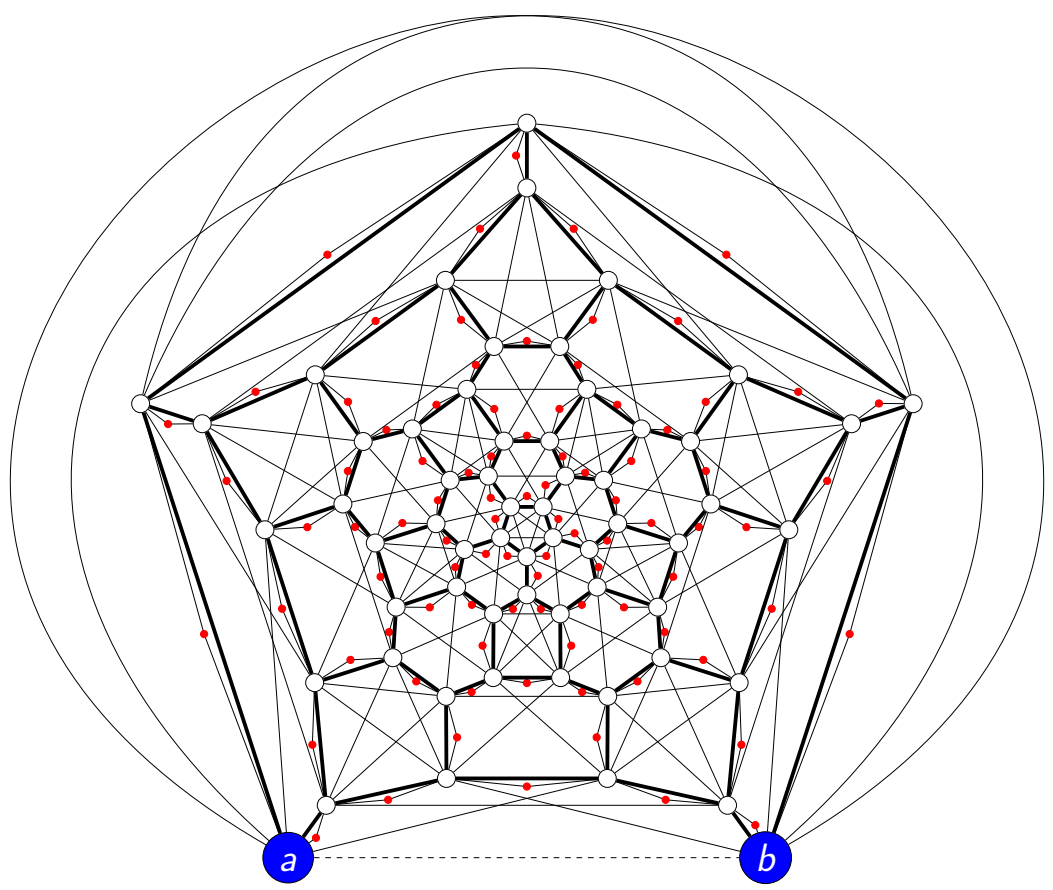

Fig. 1. Crossed Fullerene $X C_{60}$

from the planar fullerene $C_{60}$, where all pentagons are completed to $K_{5}$ s and all hexagons are crossed by three diagonals. The maximality of the $X C_{60}$ is testified by the given drawing since this graph has a unique 2-planar embedding. Then 89 hermits of degree 2 are inserted. For large $n$, take copies where the a-vertices and the b-vertices are identified each. If the rotation system is fixed, we can employ hermits of degree 1 , which leads to even lower densities.

We have explored some more interesting facts about 2-planar graphs: The $K_{7}$ is 2-planar and not 1-planar, whereas the $K_{9}$ is not 2-planar. Bipartite 2-planar graphs have at most $4 n-8$ edges. There are faces of embedded 2-planar graphs without vertices in the boundary. For example, the inner and the outer face of the $X C_{60}$ consist solely of edge segments. Moreover, there are unconnected maximal 2-planar graphs. Last, but not least, it is NPhard to test whether a graph is 2-planar even if it is given with a rotation system.

We thank Stephen Kobourov for inspiring discussions.

\section{References}

1. Brandenburg, F.J., Eppstein, D., Gleißner, A., Goodrich, M.T., Hanauer, K., Reislhuber, J.: On the Density of Maximal 1-Planar Graphs. In: Didimo, W., Patrignani, M. (eds.) GD 2012. LNCS, vol. 7704, pp. 327-338. Springer, Heidelberg (2013) 